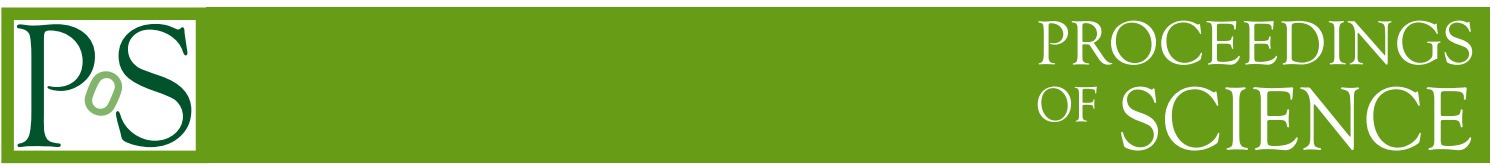

\title{
Study of tau-neutrino production at CERN SPS
}

\section{Tomoko Ariga* for the DsTau collaboration}

Kyushu University and University of Bern

E-mail: arigadartsci.kyushu-u.ac.jp

\begin{abstract}
Lepton universality in tau-neutrino $\left(v_{\tau}\right)$ scattering has been poorly tested. More precise measurement of the $v_{\tau}$ cross section would enable a search for new physics effects in $v_{\tau}$-nucleon CC interactions. Such measurement also has practical importance for next-generation neutrino oscillation experiments and astrophysical $v_{\tau}$ observations. The DsTau project has been proposed at CERN SPS to study tau-neutrino production with the aim of providing important data for future $v_{\tau}$ measurements. For $v_{\tau}$ cross section measurements, the practical method of producing an artificial $v_{\tau}$ beam employs the sequential decay of $D_{s}$ mesons produced in high-energy proton interactions. However, there has been no experimental measurement of the $D_{s}$ differential production cross section in fixed-target experiments using proton beams, which leads to a large systematic uncertainty on the $v_{\tau}$ flux estimation. The DsTau project aims to reduce this uncertainty in the current cross section measurement from about $50 \%$ to $10 \%$ by measuring the $D_{s}$ differential production cross section. For this purpose, emulsion detectors with a 50-nm spatial resolution will allow the detection of $D_{s} \rightarrow \tau \rightarrow X$ double kinks in a few mm range. Results from the beam tests conducted in 2016-2017 are presented together with a prospect for the pilot run in 2018 and the physics run in 2021.
\end{abstract}

The 39th International Conference on High Energy Physics (ICHEP2018)

4-11 July, 2018

Seoul, Korea

${ }^{*}$ Speaker. 


\section{Physics motivations}

To date, lepton universality in tau-neutrino $\left(v_{\tau}\right)$ scattering has been poorly tested. More precise measurement of the $v_{\tau}$ cross section would enable a search for new physics effects in $v_{\tau}$-nucleon $\mathrm{CC}$ interactions. Such measurement also has practical importance and value for future neutrinooscillation experiments and astrophysical $v_{\tau}$ observations. DsTau is a new project, which has been proposed at CERN SPS, to study tau-neutrino production [1] aiming at providing important data for future studies of $v_{\tau}$ interaction.

For a $v_{\tau}$ cross section measurement, the practical method of producing an artificial $v_{\tau}$ beam employs the sequential decay of $D_{s}$ mesons produced in high-energy proton interactions (Fig. 1). However, there has yet to be an experimental measurement of the $D_{s}$ differential production cross section in fixed-target experiments using proton beams, which has led to a large systematic uncertainty on the $v_{\tau}$ flux estimation. Therefore the $D_{s}$ differential production cross section must be measured as a first step towards a more precise $v_{\tau}$ cross section measurement. This was the primary source of the $>50 \%$ error in the cross section measurement by DONUT [2]. The statistical uncertainty, $33 \%$ in DONUT, is expected to be reduced to about $2 \%$ in future $v_{\tau}$ programs such as the proposed SHiP project [3].

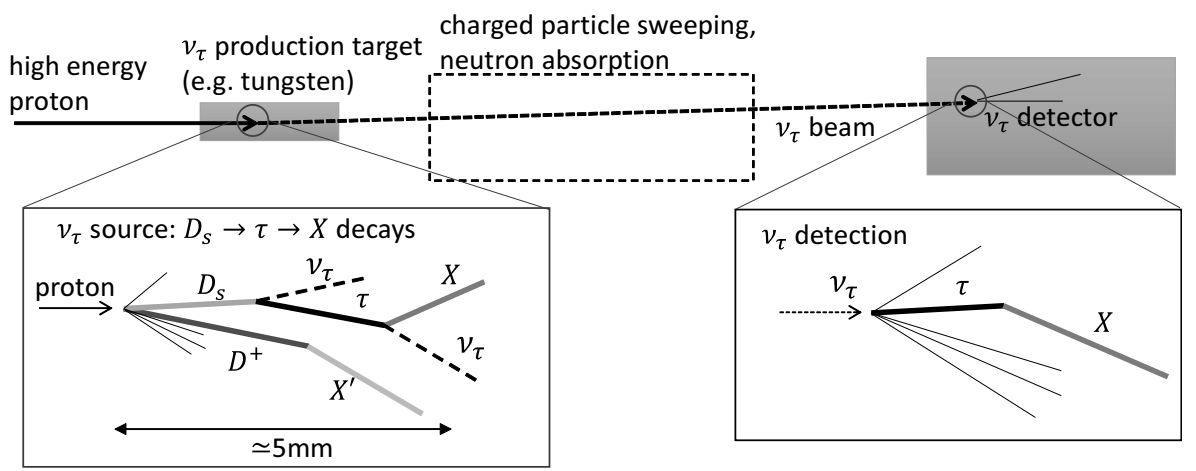

Figure 1: Concept of $v_{\tau}$ cross section measurement.

In addition to the primary objective of measuring $D_{s}$ production, analyzing $10^{8}$ proton interactions combined with the high yield of $10^{5}$ charmed decays produced as by-products will enable the extraction of additional physical quantities, including the intrinsic charm content of a proton, the interaction length of charmed hadrons, the $\Lambda_{c}$ production rates, and the search for super nuclei.

\section{Principle of the experiment}

The objective of the DsTau project is to reduce the systematic uncertainty in the cross section measurement from about $50 \%$ to $10 \%$. This will be achieved by detecting $1000 D_{s} \rightarrow \tau \rightarrow X$ events and thereby measuring the $D_{s}$ differential production cross section in $400-\mathrm{GeV}$ proton interactions. This double decay occurs at a distance of $\sim 5 \mathrm{~mm}$. The challenge of this measurement is the detection of the tiny kink angle of the $D_{s} \rightarrow \tau$ decay, which has a mean value of $7 \mathrm{mrad}$. To accomplish this, emulsion detectors with nanometric precision readout will be used. The emulsion detector has a position resolution of $50 \mathrm{~nm}$ [4], which translates to an intrinsic angular resolution 
of $0.35 \mathrm{mrad}$ with a $200-\mu \mathrm{m}$-thick plastic base layer (Fig. 2 (left)). As shown in Fig. 2 (right), each detector unit comprises a $500 \mu \mathrm{m}$-thick tungsten target, which is followed by 10 emulsion films interleaved with $200-\mu$ m-thick plastic sheets acting as high-precision particle trackers and decay volumes for short-lived particles. A module comprises ten such units followed by an ECC to measure the momenta of the daughter particles. A total of 370 modules will be exposed to a $400-\mathrm{GeV}$ proton beam from SPS at a uniform density of $10^{5}$ protons $/ \mathrm{cm}^{2}$ on the module surface. An estimated $4.6 \times 10^{9}$ protons on target will be collected, yielding $2.3 \times 10^{8}$ proton interactions in the tungsten plates, and 1000 detected $D_{s} \rightarrow \tau$ decays.
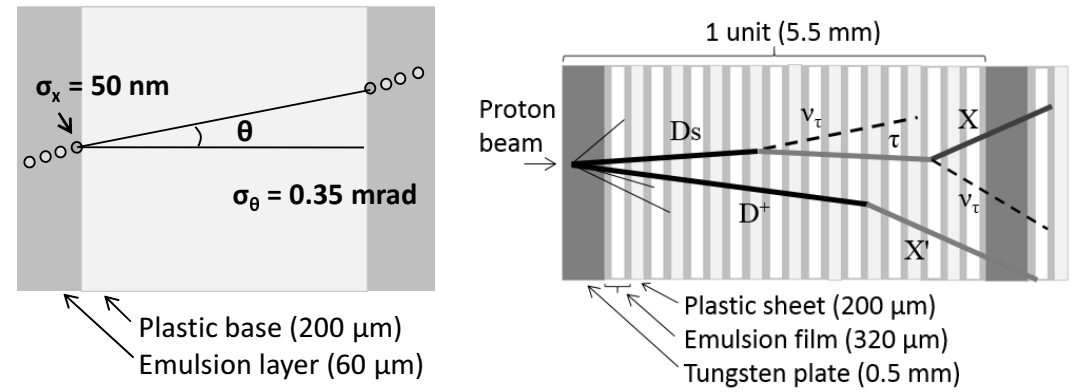

Figure 2: Left: Schematic of the angular measurement in an emulsion film. An angular precision of 0.35 mrad can be achieved by a single emulsion film. Right: A schematic of the topology of $D_{s} \rightarrow \tau \rightarrow X$ double-kink events in the detector.

\section{Readout and data analysis scheme}

The data analysis will require the full-area scanning of the 1000- $m^{2}$ emulsion surface by the world's fastest readout system, the Hyper Track Selector (HTS) [5]. After detecting $\tau$-decay topologies, events will be analyzed by dedicated high-precision systems using a piezo-based highprecision Z-axis, allowing emulsion hits to be measured with a nanometric resolution.

To study the differential production cross section of $D_{s}$ mesons, the momentum of the $D_{s}$ meson $\left(P_{D_{s}}\right)$ must be measured. Because $D_{s}$ mesons decay quickly and the invisible $v_{\tau}$ 's escape measurement, the direct measurement of $P_{D_{s}}$ is not possible. However, the peculiar event topology gives us indications of $P_{D_{s}}$. Since the $D_{s} \rightarrow \tau \rightarrow X$ decay topology has two kink angles $\left(\theta_{D_{s} \rightarrow \tau}\right.$, $\left.\theta_{\tau \rightarrow X}\right)$ and two flight lengths $\left(F L_{D_{s}}, F L_{\tau}\right)$, the combination of these four variables effectively provides an estimate of $P_{D_{s}}$. A machine learning algorithm was trained with a simulated sample $(\tau \rightarrow$ 1 prong) using the four variables to estimate $P_{D_{s}}$. The momentum resolution is estimated to be $18 \%$.

\section{Beam tests and prospect of future runs}

Two test beam campaigns were performed in November 2016 and May 2017 at CERN SPS. Figure 3 (left) shows the detector setup at the $\mathrm{H} 4$ beamline. To analyze the data, a new tracking algorithm was developed to reconstruct tracks in the extremely high track density of $\mathrm{O}\left(10^{5}-10^{6}\right)$ protons $/ \mathrm{cm}^{2}$, which is 1000 times higher than that of OPERA. An example of the reconstructed 
data from the detector is shown in Figure 3 (center), proving that analyses of short-lived particles are possible (Figure 3 (right)).
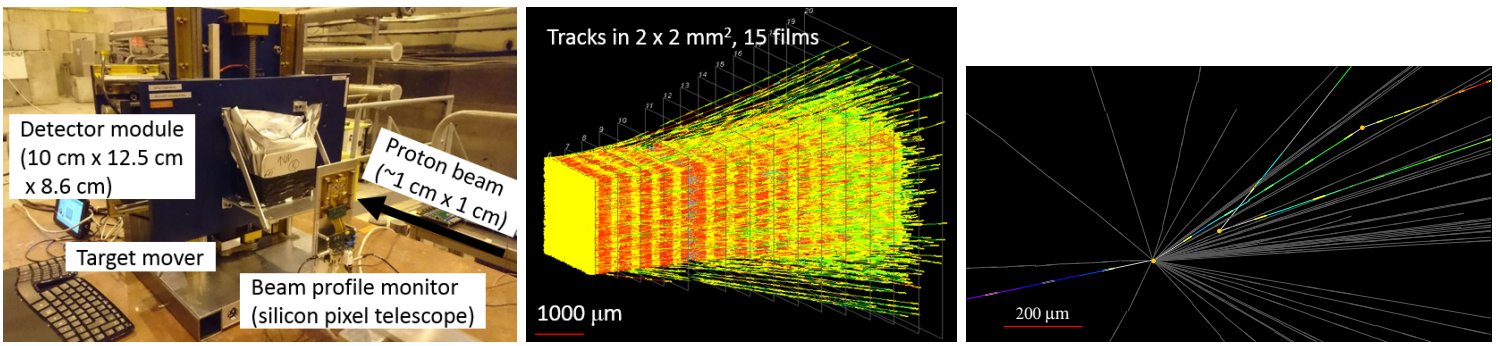

Figure 3: Left: Photo of the detector setup for the test beam campaign at the CERN SPS H4 beamline. The detector module was driven by a target stage such that it was uniformly exposed to the proton beam at a density of $10^{5}$ protons $/ \mathrm{cm}^{2}$. Center: An example of the track data reconstructed in $2 \times 2 \mathrm{~mm}^{2}$ and 15 films. About 15,000 tracks are reconstructed in this volume. Right: An event with a neutral 2-prong (vee) and a charged 1-prong (kink) topology (tilted view).

The production of emulsion films for 30 detector modules is in progress for the pilot run in August 2018. This is to test large-scale data taking and provide an estimation of the background; however, it also already allows us to re-evaluate the $v_{\tau}$ cross section measured by DONUT by significantly reducing the overall systematic uncertainty. With the outcome of the physics run in 2021 (Figure 4), DsTau will provide essential inputs for future $v_{\tau}$ experiments and pave a way for the search of new physics effects in $v_{\tau}$-nucleon CC interactions.

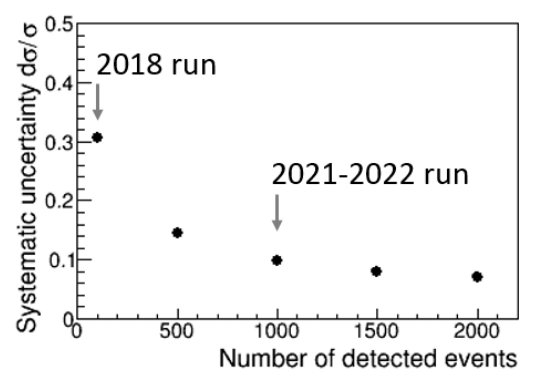

Figure 4: Expected performance of the experiment to reduce systematic uncertainty for $v_{\tau}$ cross section measurement.

\section{References}

[1] S. Aoki et al. [DsTau collaboration], CERN-SPSC-2017-029, SPSC-P-354 (2017), https://cds.cern.ch/record/2281295?ln=ja\#.

[2] K. Kodama et al., Phys. Rev. D 78 (2008) 052002.

[3] M. Anelli et al., CERN-SPSC-2015-016, SPSC-P-350 (2015).

[4] C. Amsler et al., JINST 8 (2013) P02015.

[5] M. Yoshimoto, T. Nakano, R. Komatani and H. Kawahara, PTEP 10 (2017) 103. 